\title{
In silico analysis of the molecular machinery underlying aqueous humor production: potential implications for glaucoma
}

\author{
Sarah F Janssen ${ }^{1}$, Theo GMF Gorgels ${ }^{1}$, Peter J van der Spek ${ }^{2}$, Nomdo M Jansonius ${ }^{3}$ and Arthur AB Bergen ${ }^{1,4,5^{*}}$
}

\begin{abstract}
Background: The ciliary body epithelia (CBE) of the eye produce the aqueous humor (AH). The equilibrium between the AH production by the CBE and the outflow through the trabecular meshwork ultimately determines the intraocular pressure (IOP). An increased IOP is a major risk factor for primary open angle glaucoma (POAG). This study aims to elucidate the molecular machinery of the most important function of the CBE: the AH production and composition, and aims to find possible new molecular clues for POAG and AH production-lowering drugs.

Methods: We performed a gene expression analysis of the non-pigmented (NPE) and pigmented epithelia (PE) of the human CBE of post mortem eyes. We used $44 \mathrm{k}$ Agilent microarrays against a common reference design. Functional annotations were performed with the Ingenuity knowledge database.

Results: We built a molecular model of AH production by combining previously published physiological data with our current genomic expression data. Next, we investigated molecular CBE transport features which might influence AH composition. These features included caveolin- and clathrin vesicle-mediated transport of large biomolecules, as well as a range of substrate specific transporters. The presence of these transporters implies that, for example, immunoglobins, thyroid hormone, prostaglandins, cholesterol and vitamins can be secreted by the CBE along with the AH. In silico, we predicted some of the molecular apical interactions between the NPE and PE, the side where the two folded epithelia face each other. Finally, we found high expression of seven POAG disease genes in the plasma membrane of extracellular space of the CBE, namely APOE, CAV1, COL8A2, EDNRA, FBN1, RFTN1 and TLR4 and we found possible new targets for $\mathrm{AH}$ lowering drugs in the $\mathrm{AH}$.
\end{abstract}

Conclusions: The CBE expresses many transporters, which account for AH production and/or composition. Some of these entries have also been associated with POAG. We hypothesize that the CBE may play a more prominent role than currently thought in the pathogenesis of POAG, for example by changing the composition of $\mathrm{AH}$.

Keywords: Ciliary body epithelia, Transport, Aqueous humor production, Glaucoma, Drugs

\section{Background}

The ciliary body epithelia (CBE) in the eye, consisting of the non-pigmented (NPE) and pigmented epithelia (PE), are responsible for the production of the aqueous humor $(\mathrm{AH})$. This production includes passive diffusion, active transport and production of molecules from the CBE into

\footnotetext{
* Correspondence: a.bergen@nin.knaw.nl

'Department of Clinical and Molecular Ophthalmogenetics the Netherlands Institute for Neuroscience (NIN), Royal Netherlands Academy of Arts and Sciences (KNAW), Amsterdam, the Netherlands

${ }^{4}$ Department of Ophthalmology Academic Medical Center, Amsterdam, the Netherlands

Full list of author information is available at the end of the article
}

the $\mathrm{AH}$. Via the anterior chamber, the $\mathrm{AH}$ exits the eye trough the trabecular meshwork (TM) and the canal of Schlemm into the venous blood system. The balance between the production and outflow of AH ultimately determines the intraocular pressure (IOP).

In many physiological in vitro and in vivo animal studies, the major ion-channel and transporter proteins involved in $\mathrm{AH}$ production have already been identified. In short, the chain of events leading to $\mathrm{AH}$ production starts with the PE cells that actively take up ions at their stromal (basolateral) surface (major channels $\mathrm{Na}^{+}-\mathrm{K}^{+}-2 \mathrm{Cl}^{-}$symporters, $\mathrm{Cl}^{-}-\mathrm{HCO}_{3}^{-}$and $\mathrm{Na}^{+}-\mathrm{H}^{+}$antiporters, $\mathrm{K}^{+}$channels). 
These ions flow passively through gap junctions between the PE and NPE cell. The NPE cells actively secrete ions into the $\mathrm{AH}$ (major channels $\mathrm{Na}^{+}-\mathrm{K}^{+}$-activated ATPase, $\mathrm{H}^{+}$-ATPase, $\mathrm{Na}^{+}-\mathrm{K}^{+}-2 \mathrm{Cl}^{-}$symport, $\mathrm{K}^{+}$and $\mathrm{Cl}^{-}$channels). Water follows by passive diffusion through water channels (aquaporins). Together, the studies on ion-channels led to a physiological model of $\mathrm{AH}$ production in the $\mathrm{CBE}$ (reviewed in $[1,2]$ ). However, the specific genes that code for these ion channels are still unknown for many of these channels. The first aim of our current study was to investigate the human molecular background of the physiological AH production model.

Besides the ion-channels directly involved in $\mathrm{AH}$ production, the CBE expresses also other transporters $[3,4]$. These transporters may play a role in the composition of the $\mathrm{AH}$, which determines a range of other functionalities previously assigned to the CBE. These are, for example, nourishment of avascular tissues in the eye (lens, cornea), neuro-endocrine signaling to the TM and other tissues and maintenance of the immune privilege of the eye. Our second aim of this study was to investigate the genetic identity of these transporters in the CBE.

The NPE and PE are folded, polarized neuroepithelial cell layers that face each other at their apical sides. At this side of the NPE and PE, secreted and plasma membrane biomolecules of both epithelia can interact. These biomolecules include signaling molecules, growth factors and extracellular matrix molecules. We are interested in the molecular characteristics of this apical interaction between NPE and PE.

In disease state, like primary open angle glaucoma (POAG), AH composition may be disturbed [5-7]. POAG is a neurodegenerative disease characterized by abnormal cupping of the optic nerve head and corresponding visual field defects that cannot be explained by other diseases, and with a normal open anterior chamber of the eye [8]. A major risk factor for POAG is an increased intraocular pressure (IOP) [9,10]. Proteomic analysis of POAG AH showed an increased level of total protein content and also the presence of larger and potentially harmful molecules compared to control AH [5-7]. This changed AH composition in POAG may ultimately affect the outflow facility of the TM via intrinsic molecular and structural changes, which will result in a decreased outflow and an increased IOP. The fourth aim of our study was to look for expression of POAG disease genes in the $\mathrm{CBE}$, and we hypothesize about their potential role in the CBE in POAG when these genes are mutated.

The only proven therapy of POAG is lowering of the IOP. Drugs that lower the IOP target the CBE to decrease $\mathrm{AH}$ production or target the TM to increase AH outflow. With our newly formed hypothetical molecular model of $\mathrm{AH}$ production in the CBE (first aim of this study), we finally looked for potentially new targets to change the $\mathrm{AH}$ production with already existing drugs.

\section{Methods}

\section{Ethics statement}

This study was performed in agreement with the declaration of Helsinki on the use of human material for research. The human post-mortem donor eyes were obtained from the Corneabank, Beverwijk, the Netherlands. The Corneabank obtained permission (informed consent) from the donors for enucleation of the eyes and to use the eye for scientific purposes after removal of the cornea. All data were analyzed anonymously.

\section{Tissue sampling, RNA processing and microarray}

We performed gene expression analysis on separately laser dissected NPE $(n=7)$ and PE $(n=7)$ cells (only pars plicata of the $\mathrm{CBE}$ ) with microarray studies. For detailed description of donor eye selection, tissue sampling, RNA processing and microarray performance, we refer to our previously published paper [11]. In short, we selected seven healthy human donor eyes that were snap frozen. From $20 \mu \mathrm{m}$ cryosections, the NPE and PE were separately cut out with laser dissection microscopy (PALM Carl Zeiss, Microlaser Technologies AG, Germany). Cresyl Violet staining was used to distinguish NPE (Merck, Frankfurt, Germany, art.5235). After selection of NPE and PE cells, RNA was isolated, amplified and labeled. Microarrays were performed against common reference sample (RPE/choroid RNA), in order to compare NPE and PE and normalize the data. The total gene expression datasets can be found in Gene Expression Omnibus database (GSE37957).

\section{Data analysis I: construction of sub-datasets}

From the gene expression studies on the (N)PE, we ranked the genes by expression level and assigned percentile ranks (P) [12]. Next, we formed four sub-datasets: high expression (expression $\left.>90^{\text {th }} \mathrm{P}\right)$, moderate expression $\left(50-90^{\text {th }} \mathrm{P}\right)$, low expression $\left(10-50^{\text {th }} \mathrm{P}\right)$ and very low expression $\left(<10^{\text {th }} \mathrm{P}\right)$. This means, for example, that a gene in the high expression sub-dataset has an expression intensity that falls into the highest $10 \%$ intensity values of the microarray. We also performed a statistical comparison between NPE and $\mathrm{PE}$ and stated gene expression statistically significant different between NPE and PE when p-value $<0.01$ (correction for multiple testing; Significant Different subdataset). Finally, we determined the signature genes of NPE and PE, based on the selection criteria of fold-change $>2.5$ and $p$-value $<0.01$ between NPE and PE.

From these different gene expression analyses, we studied the molecular background of $\mathrm{AH}$ production. In previous functional and physiological studies of in vitro and in vivo animal models of the CBE, ion channels that are involved in the $\mathrm{AH}$ production were widely studied. In a review of Civan and coworkers [2], these findings are put together in a model of $\mathrm{AH}$ production. We now looked for the gene expression level (high, moderate, low and very low) 
of the genes that code for these ion-channels. We also checked whether genes were a signature gene for one epithelial layer. We listed all the gene expression information of these ion-channel coding genes in Additional file 1: Table S1 and in Table 1 we presented genes with moderate or high expression level in at least one epithelial layer.

\section{Data analysis II: molecular machinery of AH production, CBE transport and (N)PE interaction}

We further studied the molecular machinery of $\mathrm{AH}$ production, CBE transport and interaction of NPE and PE. We therefore created new sub-datasets. First, we combined the genes from the Highly Expressed NPE and PE sub-datasets $\left(>90^{\text {th }} \mathrm{P}\right)$ and the genes from the Significantly Different sub-dataset (with mean gene expression value higher than $50^{\text {th }}$ percentile), since we assumed that at least these genes are of biological importance for the (N)PE $[12,13]$. We took all Significantly Different expressed genes together, so without subdivision between NPE and $\mathrm{PE}$, since it is difficult to attribute specific biological meaning in a set of up- or down regulated genes, without a base-line, to one of two specific (N)PE layers that are both healthy and from the same person ( $\mathrm{p}$-value $<0.01$ and foldchange $>2.5$ ). Still, we believe that these differences, without attributing them to a specific epithelial layer, are of biological meaning. We therefore choose to include the highly and significant different expressed genes together for future analysis. The cell specific genes were determined with much more strict criteria and stated as signature genes. The signature genes were than specifically attributed to PE or NPE. This approach was also previously used [11]. From this pool we selected those genes that coded, according to the Ingenuity knowledge database, for either plasma membrane or extracellular secreted proteins. Obviously, the entries from this selection are most likely to be involved in transmembrane transport mechanisms and interactions between two cell types compared with genes localized in the cytoplasm or nucleus. We named this new dataset, which contained highly expressed genes in the plasma membrane or extracellular space of the CBE, the Transport sub-dataset (flow diagram in Figure 1) (total Transport sub-dataset in Additional file 2: Table S2).

\section{Data analysis III: detailed analysis of the transport sub-dataset}

We analyzed the Transport sub-dataset from five different points of view:

1) In silico hypothetical molecular model of the $\mathrm{AH}$ production: We translated the model of $\mathrm{AH}$ production from Civan and coworkers [2] into a hypothetical in silico molecular model of $\mathrm{AH}$ production based on our Transport sub-dataset. We specifically indicated the signature genes in the figure.
2) Transport mechanisms in the neuro-epithelia of the CBE: We studied all transporters and ion channels present in the Transport sub-dataset to infer possible specialized transport functions of the CBE. The signature genes of NPE or PE were specifically indicated. For conformation, we performed immunohistochemistry of two proteins of interest, caveolin-1 (CAV1) and clathrin heavy chain (CLTR). The methods of immunohistochemistry were described in detail elsewhere [11]. We used primary antibodies of Abcam (Cambridge, UK), for CAV1 a rabbit polyclonal (ab2910) and for CLTR also a rabbit polyclonal (ab21679).

3) Apical PE-NPE interaction and transport mechanisms: In order to study the possible molecular interaction PE and NPE, we selected from the Transport sub-dataset those genes which encoded proteins that are apically present in the plasma membrane or apically secreted into the extracellular space. We derived the protein localizations from the Ingenuity knowledge database and from Pubmed. We used the search term 'gene name AND (apical OR basolateral OR subcellular location OR immuno location)'. We formed two new sub-datasets, named the Apical Plasma Membrane sub-dataset and Apical Extracellular secreted sub-dataset (flow-diagram in Figure 1). With these two newly formed sub-datasets, we analyzed functional interaction by exploring possible direct connections between the entries using Ingenuity's tool 'Connect', resulting in two functional molecular networks. The first one indicating the functional interaction between apical secreted molecules in the extracellular space between NPE and $\mathrm{PE}$ and the second one indicating the transport molecules in the apical plasma membrane that are connected to the apical secreted molecules from the former network. Entries connected to themselves have a circle on top; entries that did not have a connection with another gene were removed (detailed description of Ingenuity see www.ingenuity.com and [11]). Yellow symbols indicate PE signature genes.

4) CBE transport and possible implications for POAG: Based on the list of (candidate) POAG disease genes that we recently selected (reviewed by [14]), we looked in our Transport sub-dataset for presence of these (candidate) POAG disease genes.

5) Possible pharmacological targets for $\mathrm{AH}$ production altering drugs within the CBE. Within the Transport sub-dataset we looked for genes that code for targets of known AH production lowering drugs. We also looked in the hypothetical molecular model for $\mathrm{AH}$ production that we built (first aim) for drugs that target on proteins in this model. These drugs 
Table 1 Human CBE gene expression profiles for ion channels implicated in AH production

\begin{tabular}{|c|c|c|c|c|}
\hline Ion channel & Gene name & NPE & PE & SIG \\
\hline \multirow[t]{4}{*}{ Water channel } & $A Q P 1^{*}$ & $\mathrm{H}$ & $\mathrm{H}$ & \\
\hline & $A Q P 2$ & M & M & \\
\hline & AQP5 & L & M & \\
\hline & $A Q P 11^{*}$ & M & M & \\
\hline \multirow[t]{7}{*}{$\mathrm{Na}^{+} / \mathrm{K}^{+}$ATPase } & ATP1A1 ${ }^{*}$ & $\mathrm{H}$ & $\mathrm{H}$ & \\
\hline & ATP1A2 ${ }^{*}$ & $\mathrm{H}$ & $\mathrm{H}$ & \\
\hline & ATP1A3 & M & M & \\
\hline & ATP1A4 & M & $M$ & \\
\hline & ATP1B1 ${ }^{*}$ & $\mathrm{H}$ & $\mathrm{H}$ & PE \\
\hline & ATP1B2 ${ }^{*}$ & $\mathrm{H}$ & $\mathrm{H}$ & \\
\hline & ATP1B3 ${ }^{*}$ & $\mathrm{H}$ & $\mathrm{H}$ & \\
\hline \multirow[t]{8}{*}{$\mathrm{Cl}^{-}$channel } & CLCN2 & L & M & \\
\hline & CLCN3 & M & M & \\
\hline & CLCN6 & M & M & \\
\hline & CLCN7 & M & M & \\
\hline & $\mathrm{CLICI}^{*}$ & $\mathrm{H}$ & $\mathrm{H}$ & \\
\hline & CLIC2 & L & M & \\
\hline & CLIC4 ${ }^{*}$ & $\mathrm{H}$ & $\mathrm{H}$ & \\
\hline & CLIC6 ${ }^{*}$ & M & $\mathrm{H}$ & \\
\hline \multirow[t]{2}{*}{ Gap junction } & $G J A 1^{*}$ & $\mathrm{H}$ & $\mathrm{H}$ & \\
\hline & GJA4* & M & $M$ & PE \\
\hline \multirow[t]{22}{*}{$\mathrm{K}^{+}$channel } & $K C N A 5^{*}$ & L & M & \\
\hline & KCNAB1 & M & M & \\
\hline & KCNB1 & M & M & \\
\hline & $\mathrm{KCNB2}^{*}$ & L & $M$ & $\mathrm{PE}$ \\
\hline & $\mathrm{KCND2}^{*}$ & L & M & $P E$ \\
\hline & $K_{C N D 3^{*}}$ & M & M & \\
\hline & KCNE1 & M & M & \\
\hline & KCNE3 & $\mathrm{H}$ & M & \\
\hline & KCNE4 & L & M & \\
\hline & KCNG1 & M & M & \\
\hline & KCNJ12 & M & M & \\
\hline & KCN $13^{*}$ & $\mathrm{H}$ & $\mathrm{H}$ & \\
\hline & KCNJ14 & M & M & \\
\hline & KCNJ2 & M & $M$ & \\
\hline & KCNJ $8^{*}$ & M & M & \\
\hline & KCNK1 & M & $M$ & \\
\hline & KCNK13 & $M$ & $M$ & \\
\hline & KCNK15 & $M$ & $M$ & \\
\hline & KCNK3 & $M$ & L & \\
\hline & KCNK4 & $L$ & $M$ & \\
\hline & KCNK7 & $M$ & $M$ & \\
\hline & $\mathrm{KCNQ}^{*}{ }^{*}$ & $M$ & $M$ & \\
\hline
\end{tabular}

Table 1 Human CBE gene expression profiles for ion channels implicated in AH production (Continued)

\begin{tabular}{|c|c|c|c|}
\hline & $\mathrm{KCNS3}^{*}$ & M & M \\
\hline & KCNV2 & M & M \\
\hline \multirow[t]{3}{*}{$\mathrm{Na}^{+}$channel } & $S C N 2 B$ & M & M \\
\hline & $S C N 3 B$ & M & M \\
\hline & $S C N 4 B^{*}$ & $\mathrm{H}$ & $\mathrm{H}$ \\
\hline $\mathrm{Na}^{+} / 2 \mathrm{Cl}^{-} / \mathrm{K}^{+}$co-transport & SLC12A2 & M & M \\
\hline \multirow[t]{2}{*}{$\mathrm{Cl}^{-} / \mathrm{HCO}^{-}$exchanger } & $S L C 4 A 2^{*}$ & L & M \\
\hline & SLC4A3 & M & M \\
\hline \multirow[t]{2}{*}{$\mathrm{Na}^{+} / \mathrm{HCO}^{-}$symport } & SLC4A4 & M & M \\
\hline & SLC4A7 & M & M \\
\hline \multirow[t]{3}{*}{$\mathrm{Na}^{+} / \mathrm{H}^{+}$exchanger } & SLC9A5 & M & M \\
\hline & SLC9A6 & M & $L$ \\
\hline & SLC9A9 & M & M \\
\hline
\end{tabular}

Expression profiles of genes coding for ion channels implicated in aqueous humor (AH) production in healthy human non-pigmented (NPE) or pigmented epithelium (PE) of the ciliary body. The list of ion-channels involved in AH production is from Civan et al., 2004. We ranked the genes by expression level and assigned percentile ranks (P). Next, we formed four groups: high $(\mathrm{H})$ expression (expression $>90$ th $P$ ), moderate $(M)$ expression (50-90th $P$ ), low $(L)$ expression (10-50th P) and very low (VL) expression $(<10$ th P). We also compared the NPE and PE expression data and indicated if genes were signature genes (SIG) of an epithelial cell layer ( $p$-value $<0.01$ and fold change $>2.5$ ). In this table, we only presented genes with moderate or high expression level in at least one epithelial layer. The total list of ion-channels and gene expression profiles is presented in Additional file 1: Table S1. Genes indicated with ${ }^{*}$ are also present in Transport sub-dataset and indicated in Figure 2.

might be of interest for future POAG medication strategies to alter/decreased $\mathrm{AH}$ production.

\section{Results}

1. In silico hypothetical molecular model of the $\mathrm{AH}$ production

Twenty four highly expressed genes in our Transport sub-dataset coded for ion-channels involved in AH production. Furthermore, 32 additional ion-channel coding genes were moderately expressed in the NPE and/or PE (Table 1). Of all these genes, four were signature genes of the PE compared the NPE, namely ATP1B1, GJA4, $K C N B 2$, and KCND2. We translated the model of $\mathrm{AH}$ production of Civan and coworkers [2] into a hypothetical molecular model of AH production in which we included the genes of the Transport sub-dataset (Figure 2).

\section{Transport mechanisms in the neuro-epithelia of the CBE}

To determine which CBE highly expressed genes could ultimately be involved in the composition of the $\mathrm{AH}$, we assessed the expression of all transporters and (cat-)ion channels and their specific substrate in the Transport sub-dataset. Total list is outlined in Table 2. Different groups of transporters can be distinguished. 


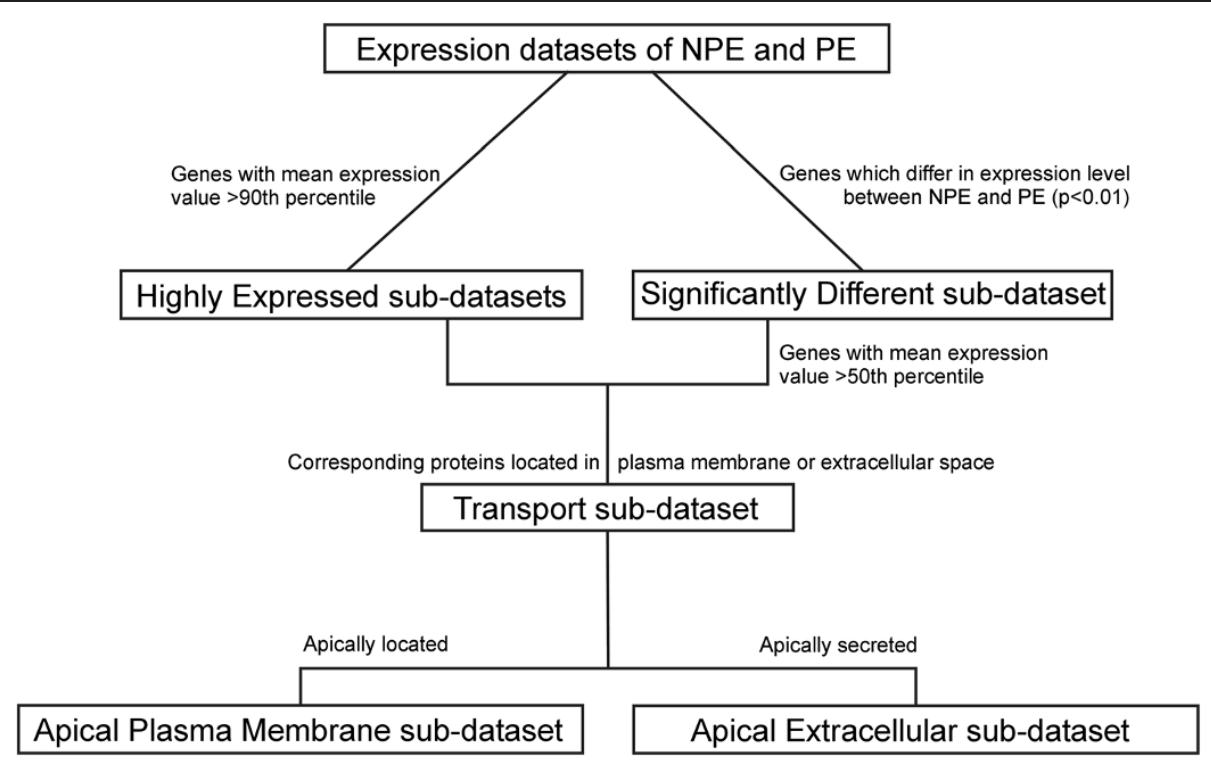

Figure 1 Flow diagram. Schematic overview of the subdivisions made in the gene expression data of the NPE and PE into different sub-datasets.

Vesicle mediated transporter gene expression in CBE: caveolin and clathrin

We identified many different entries in our Transport sub-dataset involved in endo- and exocytosis as well as vesicle mediated transport (ANXA2, -4, -5, -7, CAV1, CLTA,
CLTC, EXOC7, SORL1, STXBP3, SV2B, SYPL1, SYT13, $V A T 1$, and VTI1B).

Using immunohistochemistry, we confirmed the presence of caveolin-1 (CAV1) and clathrin heavy chain proteins (CLTR) in the CBE (Figure 3). More specifically, we found

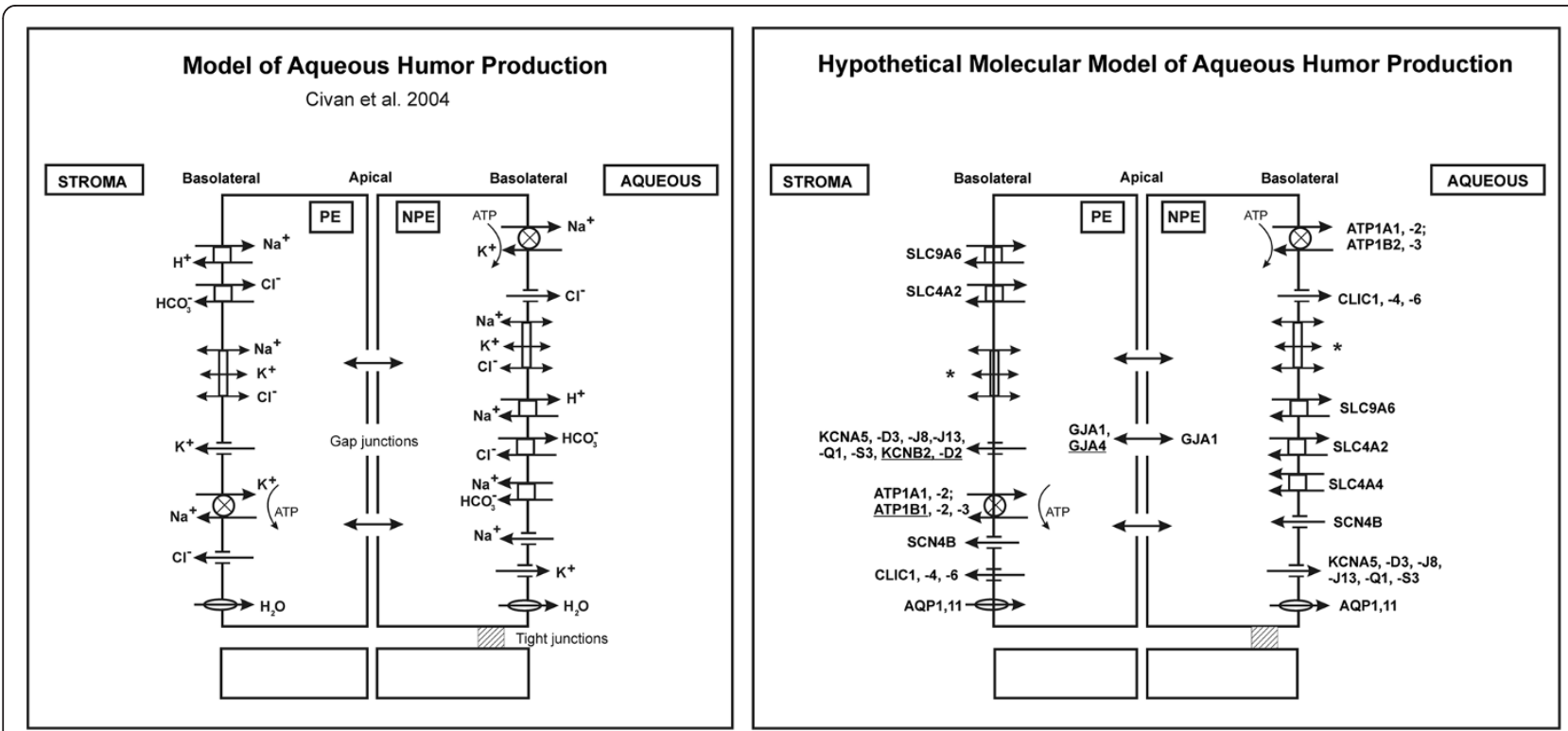

Figure 2 Hypothetical molecular model of aqueous humor production. On the left, there is the physiological model of aqueous humor (AH) production of Civan and coworkers [2]. This model includes the different ion-channels involved in the AH production. On the right, there is hypothetical in silico molecular model of AH production. This model is based on our gene expression data of the ciliary body epithelia (CBE) and our strict selection criteria of the $10 \%$ highest and significant different expressed genes of the non-pigmented (NPE) and pigmented epithelia (PE) (all together in the Transport sub-dataset; see Methods). For the $\mathrm{Na}^{+} / \mathrm{K}^{+} / 2 \mathrm{Cl}$ symporter (coded by SLC12A1 and SLC12A2) we did not found a coding gene in the Transport sub-dataset (indicated with * in the model). Genes coding for ion-channels with moderate expression levels (for example SLC12A2) can be found in Table 1. The underlined genes (ATP1B1, GJA4, KCNB2, KCND2) are signature genes of the PE compared to the NPE (fold change $>2.5$ and $p$-value $<0.01$; see Methods section). 
Table 2 All transporters in the Transport sub-dataset of the ciliary body epithelia

\begin{tabular}{|c|c|c|c|c|c|}
\hline Symbol & ID & Symbol & ID & Symbol & ID \\
\hline$\overline{\mathrm{A} 2 \mathrm{M}}$ & NM_000014 & $\mathrm{GJA} 4^{\mathrm{a}}$ & NM_002060 & SLC22A4 & NM_003059 \\
\hline$A B C A 1^{a}$ & NM_005502 & GPIHBP1 & NM_178172 & SLC22A5 & NM_003060 \\
\hline ABCA4 & NM_000350 & GPM6A & NM_201591 & $\operatorname{SLC} 23 \mathrm{~A} 2^{\mathrm{a}}$ & NM_203327 \\
\hline ABCA5 & NM_018672 & GRIN2C & NM_000835 & SLC24A3 & NM_020689 \\
\hline $\mathrm{ABCA7}$ & NM_019112 & GRIN3A & NM_133445 & $\mathrm{SLC} 26 \mathrm{~A} 7^{\mathrm{a}}$ & NM_052832 \\
\hline $\mathrm{ABCB} 1$ & NM_000927 & HCN2 & NM_001194 & SLC27A1 & NM_198580 \\
\hline$A B C C 1^{a}$ & NM_019862 & HEPH & NM_014799 & SLC29A4 & NM_001040661 \\
\hline $\mathrm{ABCC} 5$ & NM_005688 & IGFBP7 & NM_001553 & SLC2A1 & NM_006516 \\
\hline ABCC9 & NM_020297 & ITGAV ${ }^{a}$ & NM_002210 & SLC2A4 & NM_001042 \\
\hline ABCG2 & NM_004827 & KCNA5 & NM_002234 & SLC31A2 & NM_001860 \\
\hline ANO2 & NM_020373 & $\mathrm{KCNB2}^{\mathrm{a}}$ & NM_004770 & SLC35G1 & NM_153226 \\
\hline ANO6 & NM_001025356 & $\mathrm{KCND} 2^{\mathrm{a}}$ & NM_012281 & SLC38A1 & NM_030674 \\
\hline ANXA2 & NM_001002857 & KCND3 & ENST00000369697 & SLC38A2 & NM_018976 \\
\hline ANXA4 & NM_001153 & KCNJ13 & NM_002242 & SLC38A9 & NM_173514 \\
\hline ANXA5 & NM_001154 & KCNJ8 & NM_004982 & SLC39A14 & NM_015359 \\
\hline ANXA7 & NM_004034 & KCNMB4 & NM_014505 & SLC39A8 & NM_022154 \\
\hline APOE & NM_000041 & KCNQ1 & NM_000218 & SLC3A2 & NM_001012661 \\
\hline AQP1 & NM_198098 & KCNS3 & NM_002252 & $\mathrm{SLC} 40 \mathrm{~A} 1^{\mathrm{a}}$ & NM_014585 \\
\hline AQP11 & NM_173039 & LDLR & NM_000527 & SLC43A3 & NM_199329 \\
\hline ATP13A1 & NM_020410 & MAL & NM_002371 & SLC45A2 & NM_016180 \\
\hline ATP13A3 & ENST00000310773 & NRXN3 & NM_004796 & SLC4A4 & NM_003759 \\
\hline ATP1A1 & NM_000701 & ORAI1 & NM_032790 & SLC5A6 & NM_021095 \\
\hline ATP1A2 & NM_000702 & PDPN & NM_006474 & SLC6A13 & BC020867 \\
\hline $\mathrm{ATP} 1 \mathrm{~B} 1^{\mathrm{a}}$ & NM_001677 & PKD2 & NM_000297 & $\mathrm{SLC} 6 \mathrm{~A} 15^{\mathrm{a}}$ & NM_182767 \\
\hline ATP1B2 & NM_001678 & $\mathrm{RAMP}^{\mathrm{a}}$ & NM_005855 & SLC6A20 & NM_020208 \\
\hline ATP1B3 & NM_001679 & RBP1 & NM_002899 & SLC7A5 & NM_003486 \\
\hline ATP2B3 & NM_021949 & RBP4 & NM_006744 & SLC7A7 & NM_003982 \\
\hline ATP2B4 & NM_001001396 & REEP5 & NM_005669 & SLC7A8 & NM_182728 \\
\hline ATP9A & NM_006045 & SCARB1 & NM_005505 & SLC9A3R1 & NM_004252 \\
\hline BEST1 & NM_004183 & SCN4B & NM_174934 & SLC9A6 & NM_001042537 \\
\hline CACNA1C & NM_000719 & SCN8A & AK091315 & SORL1 & NM_003105 \\
\hline CAV1 & NM_001753 & SERINC1 & NM_020755 & STXBP3 & NM_007269 \\
\hline $\mathrm{CDH} 23$ & NM_022124 & SLC12A4 & NM_005072 & SV2B & NM_014848 \\
\hline CLIC4 & NM_013943 & SLC12A7 & NM_006598 & SYPL1 & NM_182715 \\
\hline CLIC6 & NM_053277 & SLC13A3 & NM_001011554 & SYT13 & NM_020826 \\
\hline CLNS1A & NM_001293 & SLC13A4 & NM_012450 & TF & NM_001063 \\
\hline CLTA & NM_007096 & SLC16A2 & NM_006517 & TM9SF2 & NM_004800 \\
\hline CLTC & NM_004859 & SLC16A3 & NM_001042422 & TNFAIP1 & NM_021137 \\
\hline CNGB3 & NM_019098 & SLC16A6 & NM_004694 & TPCN1 & AB032995 \\
\hline ECM1 & NM_004425 & SLC19A2 & NM_006996 & TRAK2 & NM_015049 \\
\hline EXOC7 & NM_001013839 & SLC1A3 & NM_004172 & TRPM1 & NM_002420 \\
\hline FOLR1 & NM_016725 & SLC1A4 & NM_003038 & TRPM3 & NM_206948 \\
\hline
\end{tabular}


Table 2 All transporters in the Transport sub-dataset of the ciliary body epithelia (Continued)

\begin{tabular}{lllll}
\hline FXYD6 $^{a}$ & NM_022003 & SLC1A7 & NM_006671 & TTR \\
GABRR1 & NM_002042 & SLC20A1 & NM_005415 & VAT1 \\
GJA1 & NM_000165 & SLC20A2 & NM_006749 & VTI1B \\
\hline
\end{tabular}

Legend: Transport sub-dataset of the CBE contains all NPE and PE highly expressed and significantly different expressed genes, whose protein products are located in the plasma membrane or secreted in the extracellular space. Genes indicated with ${ }^{a}$ are signature genes of the PE. We did not identify signature genes of the NPE that code for transporters. Abbreviations of gene names are according to standard abbreviations used in Genbank.

strong staining of CAV1 in the NPE and possible light staining in the PE. Furthermore, we observed strong staining of CLTR in both the NPE and PE of the CBE, especially in the apical plasma membrane.

\section{Specific transporter activity in CBE: hormones, vitamins, metabolism, metal ions}

We found a number of genes coding for neuro-endocrine transporters, like glutamate transporters (SLC1A3, SLC1A4, SLC1A7), glutamate-gated ion channels (GRIN2C, GRIN3A), GABA transporter (SLC6A13) and transporters of thyroid hormone (SLC16A2, TTR), thiamine (SLC19A2), vitamin C (SLC23A2; signature gene of PE), lipids (SLC27A1, APOE, LDLR, SORL1, and SCARB1) and glucose (SLC2A1, $S L C 2 A 4)$. We also identified genes coding for transporters of zinc (SLC39A14, SLC39A8), copper (SLC31A2) and iron (SLC40A1 (signature gene of PE), TF, HEPH) in the Transport sub-dataset.

\section{Transporter activity in CBE: organic anions and ions} (other than in the hypothetical molecular model of $\mathrm{AH}$ production)

We identified high expression of several genes coding for $\mathrm{ABC}$ transporters $(A B C A 1, A B C A 4, A B C A 5, A B C A 7$, $A B C B 1, A B C C 1, A B C C 5, A B C C 9$ and $A B C G 2)$, responsible for organic anion transport. $A B C A 1$ and $A B C C 1$ are signature genes of the PE when compared to the NPE. Moreover, we found genes coding for phosphate transporters (SLC20A1, SLC20A2), carnitine transporters (SLC22A4, SLC22A5), and amino acid transporters (SLC38A1, SLC6A15 (signature gene of PE), SCL7A5, $S L C 7 A 7$, SLC7A8). Next, we found genes coding for other ATP-transporters than that were already attributed for the AH production, namely ATP2B3, ATP2B4, ATP13A1, -3, and $A T P 9 A$, and genes coding for $\mathrm{K}^{+} / \mathrm{Cl}^{-}$transporters (SLC12A4, SLC12A7) and the $\mathrm{Na}^{+} / \mathrm{K}^{+} / \mathrm{Ca}^{2+}$ exchanger (SLC26A7, signature gene of PE).
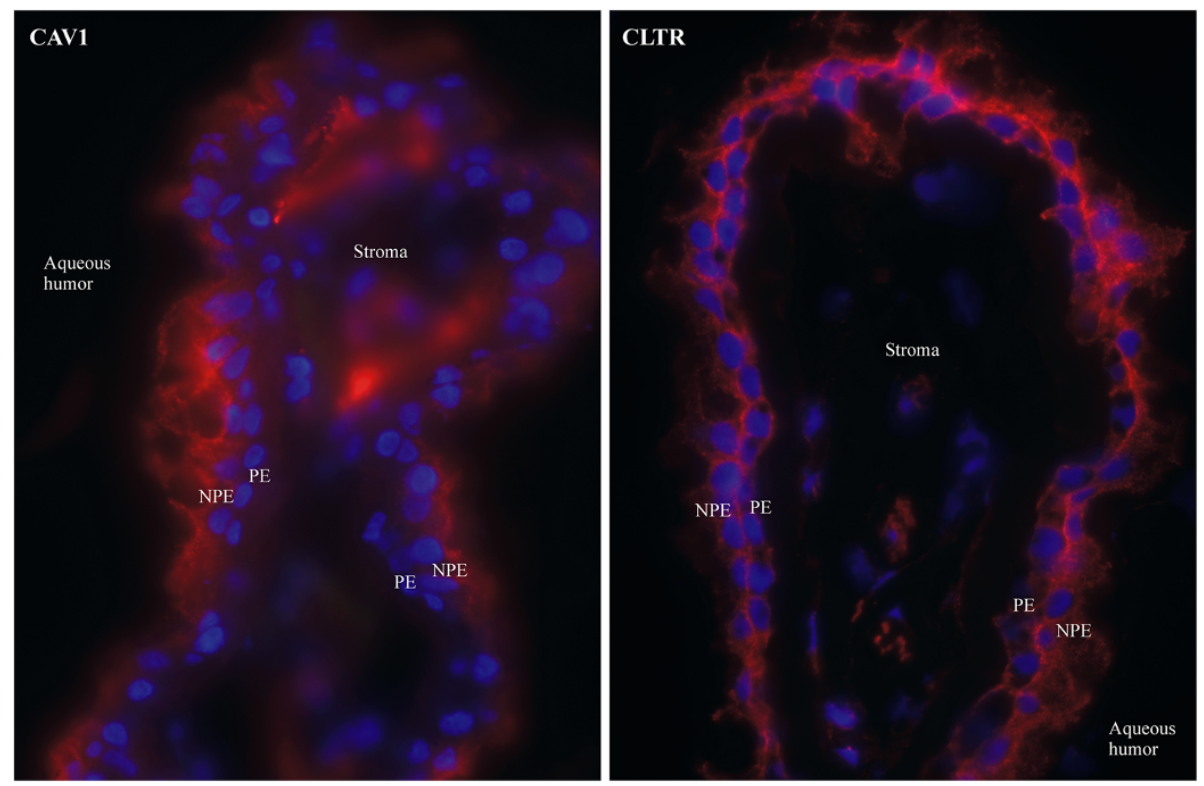

Figure 3 Immunohistochemistry of caveolin 1 and clathrin in the ciliary body. Immunofluorescence staining for caveolin 1 (CAV1) and clathrin (CLTR) in human ciliary body sections. Our gene expression data show that CAV1 is significantly different expressed between NPE and PE ( $p$-value $<0.01$ ), with higher expression in NPE and all CLTR coding genes (CLTA, CLTB and CLTC) are highly (>90 $0^{\text {th }} P$ ) expressed in both NPE and $P E$. In this immunofluorescence picture, CAV1 is clearly present in the non-pigmented epithelium (NPE) and possibly also in the pigmented epithelium (PE). We found CLTR in both NPE and PE, especially in the apical plasma membrane. Both CAV1 and CLTR were found in the vascular endothelium of the stroma. Negative controls were for both proteins negative (not shown). Legend: $b l u e=$ dapi $=$ cell nucleus; red $=c y 3=$ protein of interest. 


\section{Apical PE-NPE interaction and transport mechanisms Molecular interactions in the extracellular space between the (N)PE apical sides}

We explored the potential functional relationships between the predicted secreted entries of the Apical Extracellular Secreted sub-dataset (Figure 4). This network represents potential molecular interactions taking place in the extracellular space between the NPE and PE. The top functions assigned to this network by Ingenuity were "Neurological disease, psychological disorders and cellular movement". The network contained 34 genes of a total of 67 (51\%) secreted genes in the (N)PE apical extracellular space. Interestingly, this network contained many entries involved in matrix assembly and cytoskeleton organization (COL18A1, CTGF, DCN, FBN1, FBLN1, LTBP1, SPARC, and $S P P 1)$. Indeed, these proteins may partly fill the extracellular space between the apical membranes of the NPE and PE, as was previously shown for FBN1 by Gabriel and coworkers [15]. We also found genes coding for components of the classical complement cascade $(C 1 R$, C1S, C3, and CFB), the coagulation pathway (F10, PLAT and PROS1) and immune and/or inflammatory responses (CCL2 and IL6) and we identified many genes coding for a range of growth factors (CTGF, EGF, IGF1, IGF2, IGFBP2-6, LTBP1, PTN, TGFB2 and VEGFA). Finally, we identified several entries apparently involved in amyloid beta metabolism and plaque formation (A2M, APOE, CLU, SERPINA3, and TTR).

\section{Molecular interactions in apical transport of the $C B$}

We were interested in the transport mechanisms of the entries in the apical extracellular space of the NPE and PE. Therefore, we explored in silico potential functional relationships between the genes coding for transporters in the Apical Plasma Membrane sub-dataset and the network described above. The resulting network is displayed in Figure 5. The top functions assigned by Ingenuity to this network were "Neurological disease, psychological

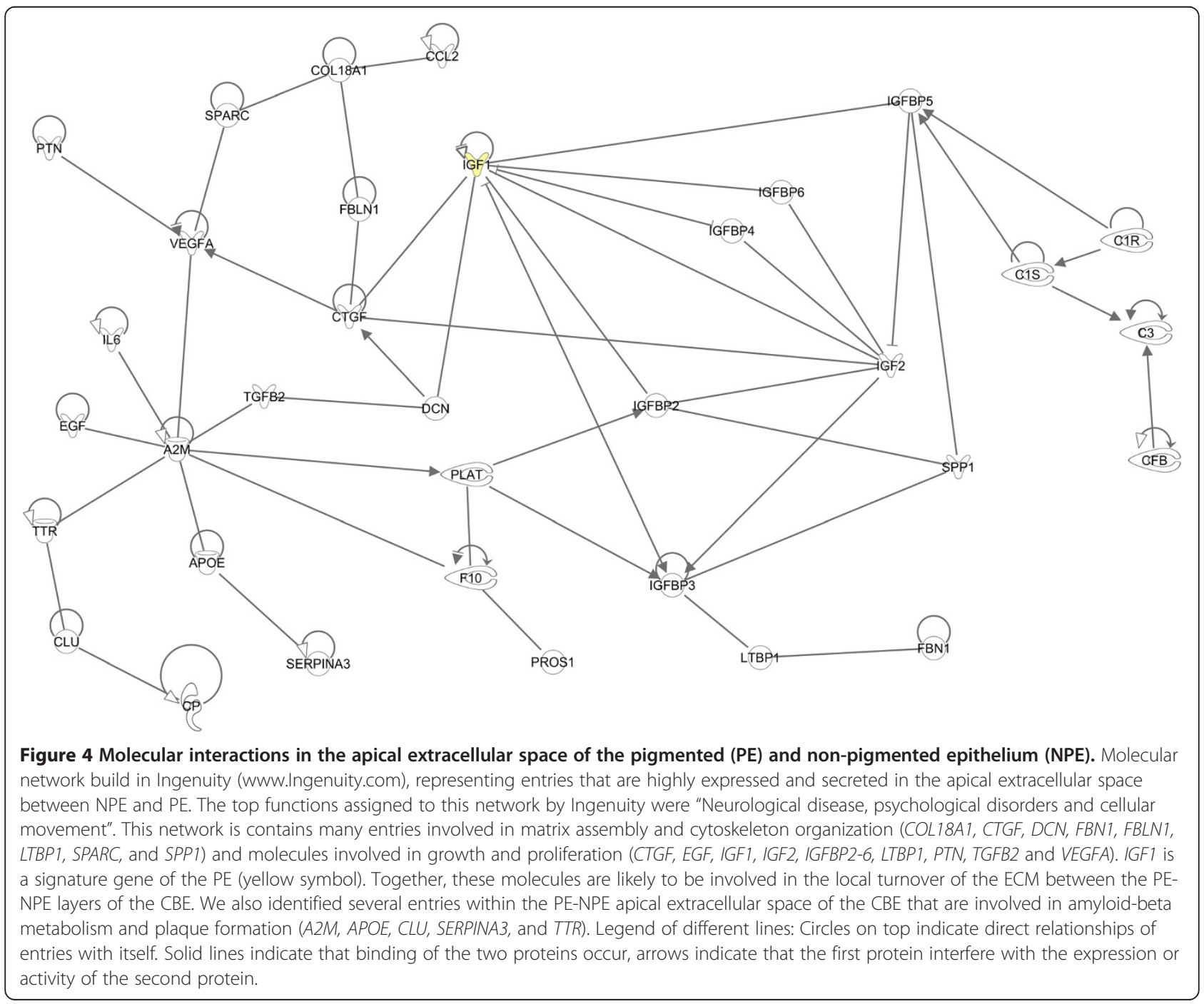




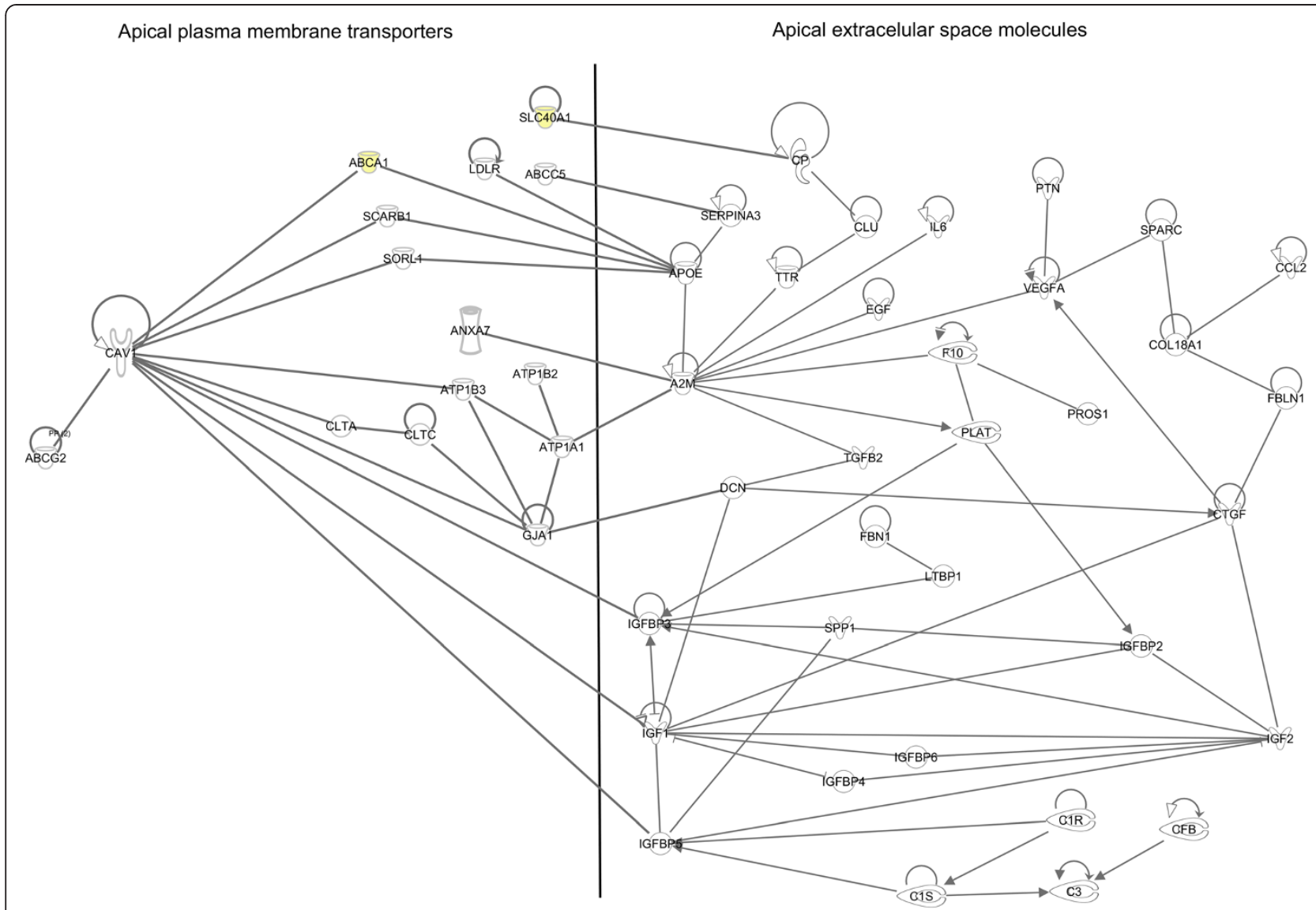

Figure 5 Molecular interactions in apical transport of the ciliary body epithelia. Molecular network build in Ingenuity (www.Ingenuity.com), representing possible transporters in the apical plasma membranes of the pigmented and non-pigmented epithelia that transport the apical extracellular excreted entries outlined in Figure 4. The top functions assigned by Ingenuity to this network were "Neurological disease, psychological disorders and cell-to-cell signaling and interaction". The major transport mechanisms to which these genes were connected were vesicle-mediated transporters (ANXA7, CAV1, CLTA, CLTC, and SORL1) and lipid (ABCA1, LDLR and SCARB1) and xenobiotic (ABCC5 and ABCG2) transporters. Two PE signature genes, $A B C A 1$ and SLC4OA1 (yellow symbols) are involved in this network. Legends of different lines: see Figure 4.

disorders and cell-to-cell signaling and interaction". This network showed direct interactions of 15 transporter genes (from a total of 50 genes; $30 \%$ ) in the apical plasma membrane with the apical extracellular secreted molecules. Clearly, several entries involved in vesicle-mediated transport were connected to the extracellular secreted entries, namely ANXA7, CAV1, CLTA, CLTC, and SORL1. Furthermore, we identified several genes in the apical plasma membrane that codes for proteins involved in lipid transport (ABCA1 (signature gene of PE), LDLR and SCARB1), iron transport (SLC4OA1; signature gene of PE) and xenobiotic/ drug transport ( $A B C C 5$ and $A B C G 2$ ) that are connected to molecules in the apical extracellular space of the CBE.

\section{CBE transport and possible implications for POAG}

In our Transport sub-dataset we found seven genes that were previously associated with POAG, namely $A P O E$, CAV1, COL8A2, EDNRA, FBN1, RFTN1 and TLR4. None of these genes were signature genes of the NPE or PE.

\section{Possible pharmacological targets for $\mathrm{AH}$ production altering drugs within the $\mathrm{CBE}$}

The three classes of drugs that can lower $\mathrm{AH}$ production are adrenergic agonists, carbonic anhydrase (CA) inhibitors and beta blockers. Within our Transport sub-dataset we found high expression of CA4 and CA14 in the plasma membrane of the CBE.

We also looked closer to the ion-channels in the hypothetical model of AH production (Figure 2) for potentially new drugs that might target these channels. For the $\mathrm{Na}+\mathrm{K}+$ ATPase (coded by ATP1A1 and ATP1A2) there are several inhibitors, namely digoxin, omeprazole, ethacrynic acid and perphenazine. Two sodium channels coded by $S C N 4 B$ and $S C N 8 A$ genes are inhibited by riluzole, a drug used in ALS. Finally, the potassium channels coded by KCNA5 and KCNQ1 can be blocked by quinidine, amiodarone, tedisamil and flecainide (antianginal and antiarrhythmic drugs) or opened by nicorandil (frequently used against hypertension). 


\section{Discussion}

In order to shed light on the molecular mechanisms in the $\mathrm{CBE}$ related to $\mathrm{AH}$ production and composition, we analyzed our expression data of the NPE and PE from five different angles. First, we studied the expression profiles of the genes coding for all ion-channels involved in $\mathrm{AH}$ production and we built in silico a hypothetical molecular model for $\mathrm{AH}$ production. Next, we assessed putative transport mechanisms in the NPE and PE, including vesicle mediated and specialized transport. Third, we analyzed, in silico, several apical molecular networks present between the NPE and PE, amongst others with respect to transport and extracellular matrix molecules. Fourth, we identified a number of clues which suggest that the CBE plays an important (transport) role in POAG. And finally, we looked for existing and possible new pharmacological targets in the $\mathrm{CBE}$ which could potentially modify the AH production.

Our microarray approach and corresponding functional annotations have several limitations, which are extensively discussed elsewhere $[12,13]$. The gene expression datasets used in the current study were first roughly analyzed in a previous paper, which describes the general properties of the CBE [11]. The value of the current study is that we now analyzed these datasets in detail, primarily focused on the molecular machinery underlying transport mechanisms. Fortunately, the physiology of the transport mechanisms in the CBE has previously already been studied extensively in in vitro and in vivo models, and was leading for our molecular analysis and interpretation.

Obviously, the amount of (combination of old and) new data presented in this manuscript prohibits verification in vitro and in vivo models in one single study, let alone in this manuscript.

Finally, it must be taken into account that we used very strict selection criteria for our Transport subdataset. Therefore, the genes that we selected for further future analysis in this manuscript may not cover all genes that are involved in the transport and $\mathrm{AH}$ production functions of the CBE. It is not unlikely that there is functional redundancy, and that several similar genes may code for more or less effective additional transport proteins, or that more - yet to be identified proteins may be involved in AH production. On the other hand, because of our strict selection criteria, the genes that are present in this model, are likely to be truly involved in the molecular machinery of $\mathrm{AH}$ production and $\mathrm{CBE}$ transport mechanisms.

\section{In silico hypothetical molecular model of the AH production}

We build a hypothetical molecular model of $\mathrm{AH}$ production based on the gene expression data of (the ion-channels in) the CBE (Figure 2). We selected genes under strict selection criteria in the Transport sub-dataset.

Due to these strict selection criteria, a number of additional genes or proteins, previously implicated in $\mathrm{AH}$ production, did not pop up in our present hypothetical molecular model (Figure 2). For example, for the $\mathrm{Na}^{+} / \mathrm{K}^{+} / 2 \mathrm{Cl}^{-}$symporter (coded by $S L C 12 A 1$ or $S L C 12 A 2$ ) we did not found a coding gene in our Transport subdataset, but these genes were moderately (SLC12A2) and lowly (SLC12A1) expressed in the (N)PE (Table 1).

\section{Transport mechanisms in the neuro-epithelia of the CBE}

The CBE expresses many genes coding for ion-channels and transporters at high levels. Due to their known substrate specificity of their corresponding proteins, we can also predict the biomolecules transported by these CBE entries. Consequently, our inventory, in combination with data from the literature, showed that the $\mathrm{CBE}$ most likely transports a wide variety of molecules, including cations, organic cations and anions, amino acids, glutamate, GABA, iron, copper, zinc, thyroid hormone, vitamin A, $B$ and $C$, glucose, and fatty acids. Indeed, the majority of these ions and biomolecules were also found in previous proteomic studies of the $\mathrm{AH}$, which suggests that the $\mathrm{CBE}$ is an important ocular entry point of these molecules [16-20].

Interestingly, we also observed high expression of many entries involved in vesicle mediated trans- or endocytosis. For example, we found high expression of CAV1 and $C L T C$ in the CBE and we confirmed their presence by immunohistochemistry (Figure 3). Caveolin and clathrin are involved in vesicle mediated endo-, exo- and transcytosis of large molecules through the cell [21-25]. Caveolinmediated vesicles transport for example iron, transferrin, insulin, lipids, albumin, chemokines and pathogens [21]. Clathrin-mediated vesicles can transport nutrients, viruses, toxins, plasma membrane proteins and signaling receptors, including $ß 2$-adrenergic receptors, CD4, insulin receptors, T-cell and B-cell receptors [24,25]. While caveolin transport is more or less a-specific, clathrin-mediated vesicle transport is only possible with adaptor proteins (receptors). We also found expression of several genes coding for other proteins involved in less specific vesicle transport, like ANXA2, EXOC7, SORL1, STXBP3, SYT13, VAT1, and VTI1B

In summary, our data, in combination with the literature, suggest that both general and specific vesicle mediated transport play a role in the transport of a range of biomolecules over the CBE neuroepithelia. Indeed, this was already suggested by a histochemical electron microscope study [26]. We hypothesize that these transport mechanisms may (also) be involved in $\mathrm{AH}$ composition, in particular with regard to the presence of large molecules. 


\section{Apical PE-NPE interaction and transport mechanisms}

In silico, we assigned several interacting proteins to the extracellular space between the PE and NPE, where transported molecules from the blood to the $\mathrm{AH}$ are likely to pass. We found several proteins involved matrix assembly and cytoskeleton organization, a network of protease (inhibitors) and a group of proteins involved in cell growth and proliferation pathways. These molecules are likely to be involved in the local turnover of the ECM between the PE-NPE layers of the CBE. We also identified several entries within the PE-NPE apical extracellular space of the CB that are involved in amyloid-beta metabolism and plaque formation. The major transport mechanisms to which these entries were connected were vesicle-mediated transporters and lipid and xenobiotic transporters. The molecules within the PE-NPE extracellular space and their transporters may influence intrinsic filtration or secretion capabilities of the CBE. If disrupted by a genetic mutation, or altered by age-related oxidative stress, these molecules change and may affect the $\mathrm{AH}$ secretion and composition.

\section{CBE transport and possible implications for POAG}

We identified seven POAG candidate disease genes in our Transport sub-dataset. These genes were APOE, CAV1, COL8A2, EDNRA, FBN1, RFTN1 and TLR4. We hypothesized that mutations in these genes might modify the $\mathrm{AH}$ production and composition via the CBE. Although the production rate of $\mathrm{AH}$ is hardly changed in POAG [27], the composition of $\mathrm{AH}$ does, with overall an increase of (large and harmful) proteins in POAG AH compared to controls [5-7]. The POAG disease genes that we found in our Transport sub-dataset code for structural components (FBN1, COL8A2), transporters (CAV1, APOE, RFTN1) and signaling molecules (CAV1, EDNRA), all potentially involved in $\mathrm{AH}$ production and composition. Further research is warranted to prove or reject their involvement in disturbed $\mathrm{AH}$ dynamics of the $\mathrm{CBE}$ during POAG. It would be of interest to study mouse models with mutations in these genes, measure IOP, optic nerve head characteristics and $\mathrm{AH}$ composition. It would also be of interest to study the effect of IOP-lowering drugs in animals with mutations in these target genes in comparison with controls.

\section{Possible pharmacological targets for AH production altering drugs within the $\mathrm{CBE}$}

Last but not least, we annotated our molecular model (Figure 2, discussed above) with possible new therapeutic targets that might alter AH production. First, we found several drugs that block $\mathrm{Na}+/ \mathrm{K}+$ ATPases: digoxin, ethacrynic acid, omeprazol, and perphenazine. Digoxin has antiarrhythmic effects via myocytes and some older literature sources describe the lowering effect of digoxin on the IOP in human [28-30] and cats [31], probably due to blocking of $\mathrm{Na}^{+} / \mathrm{K}^{+}$-ATPase in the CBE. Ethacrynic is a diuretic drug and multiple animal studies already showed the IOP lowering effect of intracameral or topical administered ethacrynic acid in the eye, probably through increased AH outflow, but possibly also by decreased AH production [32-39]. The major disadvantage of athacrynic acid is the risk for (severe) corneal edema. Omeprazole is a proton pump inhibitor that inhibits gastric acid secretion in the stomach. In the literature, we found one clinical study in which treatment with omeprazole indeed lowered the IOP [40]. The drug perphenazine is an antagonist of dopamine receptors and has antiemetic and antipsychotic effects. We found no studies of perhanzine in relation to IOP altering effects.

Secondly, we found one drug that block sodium channels, namely riluzole. This drug is used in ALS. In the literature, we couldn't find studies describing an effect on IOP with riluzole use.

Finally, we found several drugs that influence the activity of potassium channels. Quinidine, amiodarone, tedisamil and flecainide block potassium channels, while nicorandil opens them. Only for the latter drug, nicorandil, two studies exist on the IOP effects. Chiang and coworkers [41] found increased IOPs after nicorandil use in rabbits, whereas Chowdhury and coworkers [42] found an IOP lowering effect of nicorandil in rats.

In conclusion, we found possible new therapeutic targets for AH production modification in the CBE. These targets are of interest, since IOP lowering is currently the only proven therapy for POAG and current medical treatment modalities often fail to reduce the IOP sufficiently. More research into these new targets might result in new treatment options for POAG, which may make surgery, with its side effects and risks, less often needed. Extensive studies in animal models are warranted to study potential beneficial effects of these drugs on the IOP and their potential harmful side effects.

\section{Conclusions}

The transport mechanisms in the CBE are numerous, ranging from extensively studied specific ion channels and pumps that form the basis of $\mathrm{AH}$ production, to more generalized transport functions like vesicle mediated transport. The CBE transports (neuro-) endocrine and metabolic particles and is involved in specific apical transport of ECM proteins and growth factors. Taken together, these transport mechanisms are important in determining the $\mathrm{AH}$ production rate and composition. In POAG, the composition of the AH is changed and several POAG disease gene are highly expressed, and their corresponding proteins localized in the CBE plasma membrane or extracellular space. We propose that mutations in these POAG genes cause disturbances in the $\mathrm{AH}$ composition by the $\mathrm{CBE}$, resulting in a changed $\mathrm{AH}$ content and 
disturbed outflow facility by the TM. Finally, we found possible new therapeutic targets for $\mathrm{AH}$ production modification in the CBE.

\section{Additional files}

Additional file 1: Table S1. Total human CBE gene expression profile for AH production.

Additional file 2: Table S2. Total Transport sub-dataset.

\section{Abbreviations}

AH: Aqueous humor; CBE: Ciliary body epithelia; IOP: Intraocular pressure; NPE: Non-pigmented epithelium; PE: Pigmented epithelium; POAG: Primary open angle glaucoma; TM: Trabecular meshwork.

\section{Competing interests}

The authors declare that they have no competing interests

\section{Authors' contributions}

SFJ carried out the laboratory work, performed the bioinformatic analysis and drafted the manuscript. TGG participated in the design of the study and the manuscript. PJS provided bioinformatics software and background knowledge. NMJ participated in the design of the study and the manuscript. $A A B$ performed bioinformatic analysis and drafted the manuscript. All authors read and approved the final manuscript.

\section{Acknowledgement}

We thank the Corneabank, Beverwijk, the Netherlands, for providing donor eyes.

This study was supported by grants from the General Dutch Foundation Preventing Blindness, the National Foundation for Blinds and Low Vision, the Foundation Blinden-Penning, the Foundation Glaucoomfonds, the Rotterdam Foundation of Blinds and the Professor Mulder Foundation (all coordinated by UitZicht, project \# UitZicht2008-7). The funders had no role in study design, data collection and analysis, decision to publish, or preparation of the manuscript.

\section{Author details}

${ }^{1}$ Department of Clinical and Molecular Ophthalmogenetics the Netherlands Institute for Neuroscience (NIN), Royal Netherlands Academy of Arts and Sciences (KNAW), Amsterdam, the Netherlands. ${ }^{2}$ Department of Bioinformatics, Erasmus University Medical Center, Rotterdam, the Netherlands. ${ }^{3}$ Department of Ophthalmology, University Medical Center Groningen, University of Groningen, Groningen, the Netherlands. ${ }^{4}$ Department of Ophthalmology Academic Medical Center, Amsterdam, the Netherlands. ${ }^{5}$ Department of Clinical Genetics Academic Medical Center, Amsterdam, the Netherlands.

\section{Received: 23 September 2013 Accepted: 23 October 2013}

Published: 28 October 2013

\section{References}

1. Civan MM: Transport components and net secretion of the aqueous humor and their integrated regulation. In Current Topics in Membranes: The eye's aqueous humor. From secretion to glaucoma, Volume 45. Edited by Civan MM. San Diego, London, Boston, New York, Sydney, Tokyo, Toronto: Academic Press; 1998:1-24

2. Civan MM, Macknight AD: The ins and outs of aqueous humour secretion. Exp Eye Res 2004, 78:625-631.

3. Kraft ME, Glaeser H, Mandery K, Konig J, Auge D, Fromm MF, et al: The prostaglandin transporter OATP2A1 is expressed in human ocular tissues and transports the antiglaucoma prostanoid latanoprost. Invest Ophthalmol Vis Sci 2010, 51:2504-2511.

4. Shin BC, Suzuki T, Tanaka S, Kuraoka A, Shibata Y, Takata K: Connexin 43 and the glucose transporter, GLUT1, in the ciliary body of the rat. Histochem Cell Biol 1996, 106:209-214.
5. Duan XM, Xue P, Wang NL, Dong Z, Lu QJ, Yang FQ: Proteomic analysis of aqueous humor from patients with primary open angle glaucoma. Mol Vis 2010, 16:2839-2846.

6. Grus FH, Joachim SC, Sandmann S, Thiel U, Bruns K, Lackner KJ, et al: Transthyretin and complex protein pattern in aqueous humor of patients with primary open-angle glaucoma. Mol Vis 2008, 14:1437-1445.

7. Izzotti A, Longobardi M, Cartiglia C, Sacca SC: Proteome alterations in primary open angle glaucoma aqueous humor. J Proteome Res 2010 9:4831-4838.

8. Casson RJ, Chidlow G, Wood JP, Crowston JG, Goldberg I: Definition of glaucoma: clinical and experimental concepts. Clin Experiment Ophthalmol 2012, 40:341-349.

9. Leske MC, Connell AM, Wu SY, Hyman LG, Schachat AP: Risk factors for open-angle glaucoma. The Barbados Eye Study. Arch Ophthalmol 1995 113:918-924.

10. Weinreb RN, Khaw PT: Primary open-angle glaucoma. Lancet 2004, 363:1711-1720.

11. Janssen SF, Gorgels TG, Bossers $K$, ten Brink JB, Essing AH, Nagtegaal M, et al: Gene expression and functional annotation of the human ciliary body epithelia. Plos One 2012, 7:e44973.

12. Booij JC, van Soest S, Swagemakers SMA, Essing AHW, Verkerk AJMH, van der Spek PJ, et al: Functional annotation of the human retinal pigment epithelium transcriptome. Bmc Genomics 2009, 10:164.

13. Booij JC, ten Brink JB, Swagemakers SM, Verkerk AJ, Essing AH, van der Spek $P J$, et al: A new strategy to identify and annotate human RPE-specific gene expression. Plos One 2010, 5:e9341.

14. Janssen SF, Gorgels TGNF, Ramdas WD, Klaver CCW, Van Duijn CM, Jansonius NM, et al: The Vast Complexity of Primary Open Angle Glaucoma: Disease Genes, Risks, Molecular Mechanisms and Pathobiology. Prog Retin Eye Res 2013. in press.

15. Gabriel LA, Wang LW, Bader H, Ho JC, Majors AK, Hollyfield JG, et al: ADAMTSL4, a secreted glycoprotein widely distributed in the eye, binds fibrillin-1 microfibrils and accelerates microfibril biogenesis. Invest Ophthalmol Vis Sci 2011, 1:461-469.

16. Chowdhury UR, Madden BJ, Charlesworth MC, Fautsch MP: Proteome Analysis of Human Aqueous Humor. Invest Ophthalmol Vis Sci 2010, 51:4921-4931.

17. Cumurcu T, Mendil D, Etikan I: Levels of zinc, iron, and copper in patients with pseudoexfoliative cataract. Eur J Ophthalmol 2006, 16:548-553.

18. Kallberg ME, Brooks DE, Gelatt KN, Garcia-Sanchez GA, Szabo NJ, Lambrou GN: Endothelin-1, nitric oxide, and glutamate in the normal and glaucomatous dog eye. Vet Ophthalmol 2007, 10:46-52.

19. Leite MT, Prata TS, Kera CZ, Miranda DV, Barros SBD, Melo LAS: Ascorbic acid concentration is reduced in the secondary aqueous humour of glaucomatous patients. Clin Exp Ophthalmol 2009, 37:402-406.

20. Pelletier CC, Lambert JL, Borchert M: Determination of glucose in human aqueous humor using Raman spectroscopy and designed-solution calibration. App/ Spectrosc 2005, 59:1024-1031.

21. Frank PG, Pavlides S, Lisanti MP: Caveolae and transcytosis in endothelial cells: role in atherosclerosis. Cell Tissue Res 2009, 335:41-47.

22. Jung N, Haucke V: Clathrin-mediated endocytosis at synapses. Traffic 2007, 8:1129-1136.

23. Parkar NS, Akpa BS, Nitsche LC, Wedgewood LE, Place AT, Sverdlov MS, et al: Vesicle Formation and Endocytosis: Function, Machinery, Mechanisms, and Modeling. Antioxid Redox Signal 2009, 11:1301-1312.

24. Puertollano R: Clathrin-mediated transport: assembly required Workshop on Molecular Mechanisms of Vesicle Selectivity. Embo Report 2004, 5:942-946.

25. Tuma PL, Hubbard AL: Transcytosis: Crossing cellular barriers. Physiol Rev 2003, 83:871-932.

26. Eggli PS, Graber W, Vanderzypen E: Formation and Release of Vesicles from the Basal Surfaces of Rat Eye Nonpigmented Ciliary Epithelial-Cells - A Novel Secretory Mechanism. Anat Rec 1991, 231:156-166.

27. Larsson LI, Rettig ES, Brubaker RF: Aqueous flow in open-angle glaucoma. Arch Ophthalmol 1995, 113:283-286.

28. Hardt BW, Johnen R, Fahle M: The influence of systemic digitalis application on intraocular pressure. Graefes Arch Clin Exp Ophthalmol 1982, 219:76-79.

29. Hegazy MA, Labib MA, Ghaleb $H$, Barradah A: Effect of digoxin on intraocular pressure. Bull Ophthalmol Soc Egypt 1967, 60:83-91.

30. Peczon JD: Clinical evaluation of digitalization in glaucoma. Arch Ophthalmol 1964, 71:500-504. 
31. Ferraiolo BL, Pace DG: Digoxin-induced decrease in intraocular pressure in the cat. Eur J Pharmacol 1979, 55:19-22.

32. Arnold JJ, Choksi Y, Chen X, Shimazaki A, Hatten J, Toone EJ, et al: Eyedrops containing SA9000 prodrugs result in sustained reductions in intraocular pressure in rabbits. $J$ Ocul Pharmacol Ther 2009, 25:179-186.

33. Croft MA, Hubbard WC, Kaufman PL: Effect of ethacrynic acid on aqueous outflow dynamics in monkeys. Invest Ophthalmol Vis Sci 1994, 35:1167-1175.

34. Green K, Mayberry L: Drug effects on the hydraulic conductivity of the isolated rabbit ciliary epithelium. Q J Exp Physiol 1985, 70:271-281.

35. Searles RV, Johnson M, Shikher V, Balaban CD, Severs WB: Effects of ethacrynic acid on intraocular pressure of anesthetized rats. Proc Soc Exp Biol Med 1999, 220:184-188.

36. Shimazaki A, Ichikawa M, Rao PV, Kirihara T, Konomi K, Epstein DL, et al: Effects of the new ethacrynic acid derivative SA9000 on intraocular pressure in cats and monkeys. Biol Pharm Bull 2004, 27:1019-1024.

37. Tingey DP, Schroeder A, Epstein MP, Epstein DL: Effects of topical ethacrynic acid adducts on intraocular pressure in rabbits and monkeys. Arch Ophthalmol 1992, 110:699-702.

38. Tingey DP, Ozment RR, Schroeder A, Epstein DL: The effect of intracameral ethacrynic acid on the intraocular pressure of living monkeys. Am J Ophthalmol 1992, 113:706-711.

39. Wang RF, Podos SM, Serle JB, Lee PY, Neufeld AH, Deschenes R: Effects of topical ethacrynic acid ointment vs timolol on intraocular pressure in glaucomatous monkey eyes. Arch Ophthalmol 1994, 112:390-394.

40. Kountouras J, Mylopoulos N, Chatzopoulos D, Zavos C, Boura P, Konstas AG, et al: Eradication of Helicobacter pylori may be beneficial in the management of chronic open-angle glaucoma. Arch Intern Med 2002, 162:1237-1244.

41. Chiang CH, Lin CH: Effects of cromakalim and nicorandil on intraocular pressure after topical administration in rabbit eyes. J Ocul Pharmacol Ther 1995, 11:195-201.

42. Chowdhury UR, Bahler CK, Hann CR, Chang M, Resch ZT, Romero MF, et al: ATP-sensitive potassium (KATP) channel activation decreases intraocular pressure in the anterior chamber of the eye. Invest Ophthalmol Vis Sci 2011, 52:6435-6442.

doi:10.1186/2043-9113-3-21

Cite this article as: Janssen et al:: In silico analysis of the molecular machinery underlying aqueous humor production: potential implications for glaucoma. Journal of Clinical Bioinformatics 2013 3:21.

\section{Submit your next manuscript to BioMed Central and take full advantage of:}

- Convenient online submission

- Thorough peer review

- No space constraints or color figure charges

- Immediate publication on acceptance

- Inclusion in PubMed, CAS, Scopus and Google Scholar

- Research which is freely available for redistribution 